\title{
Los/as estudiantes marroquíes en España : migración, retorno y género
}

\section{Eva María González Barea}

\section{Q OpenEdition \\ 1 Journals}

\section{Edición electrónica}

URL: https://journals.openedition.org/remi/2030

DOI: $10.4000 /$ remi.2030

ISSN: $1777-5418$

\section{Editor}

Université de Poitiers

\section{Edición impresa}

Fecha de publicación: 1 diciembre 2004

Paginación: 147-161

ISBN: 2-911627-38-5

ISSN: 0765-0752

\section{Referencia electrónica}

Eva María González Barea, «Los/as estudiantes marroquíes en España : migración, retorno y género», Revue européenne des migrations internationales [En línea], vol. 20 - n³ | 2004, Publicado el 25 septiembre 2008, consultado el 14 abril 2022. URL: http://journals.openedition.org/remi/2030 ; DOI: https://doi.org/10.4000/remi.2030

Este documento fue generado automáticamente el 14 abril 2022.

(C) Université de Poitiers 


\title{
Los/as estudiantes marroquíes en España : migración, retorno y género
}

\author{
Eva María González Barea
}

1 El presente artículo incluye el análisis de un proceso migratorio particular y, al mismo tiempo, novedoso dentro de la perspectiva de estudio de los movimientos migratorios contemporáneos en el contexto español. Se trata del desplazamiento de los/as jóvenes marroquíes que se dirigen a España y, en concreto, a Granada para realizar estudios universitarios.

2 Este articulo se apoya sobre una investigación ${ }^{1}$ cuya relevancia radica, por una parte, en el estudio de este desplazamiento por motivos académicos desde una visión global de los movimientos migratorios ; y, por otra parte, su importancia se encuentra en el análisis del proceso migratorio a través de tres fases o etapas del mismo, denominadas respectivamente, etapa pre-migratoria, migratoria y post-migratoria, que a pesar de su supuesta temporalidad lineal configura un proceso cíclico en la migración. Este articulo enfoca la última de estas etapas.

3 El objetivo principal de este estudio ha sido el análisis del proceso migratorio de los/as estudiantes marroquíes a través de sus propios discursos. Dicho análisis ha incluido dos categorías principales : la diferencia religiosa y la estructuración de las relaciones sociales de género, como posibles vertebradores de la migración en cada una de sus etapas.

4 Dicho estudio etnográfico responde al desarrollo de un metodología cualitativa (Aguirre, 1995) que ha permitido describir incidentes clave, funcionalmente relevantes situándolos en relación con el más amplio contexto socio-cultural. Desde esta metodología de investigación se han aplicado técnicas de recogida de datos abiertas, entrevistas semi-estructuradas y observación participante, así como la realización de un muestreo intencional, es decir, la muestra no pretende representar a una población con el objetivo de generalizar los resultados. Un anexo, al final del articulo, presenta la metodología del proceso de investigación. 


\section{Factores de elección de la universidad de Granada}

Según un primer informe realizado por López García (1999), el 49,6 \% de los estudiantes de nacionalidad marroquí que se encuentran en Andalucía está instalado en la ciudad de Granada. Los últimos datos (febrero 2000), ofrecidos por la Asociación de Estudiantes Marroquíes (AEM), indican que tres mil trescientos, aproximadamente, son los estudiantes marroquíes en España, de los cuales en Granada están instalados dos mil cien, es decir, un $60 \%$ del total del Estado ${ }^{2}$. Estas cifras muestran la existencia de una realidad académica y sociocultural en la universidad, que se caracteriza por su dinámica de crecimiento, constituyendo los marroquíes la colonia más numerosa de estudiantes extranjeros.

Existen determinados factores que explican la presencia de estos/as estudiantes marroquíes en la Universidad de Granada y que justifican la realización del proceso migratorio analizado. Uno de ellos es la existencia de una historia compartida entre España y Marruecos lo cual ha facilitado la cooperación institucional en materia de educación entre dichos países, así gran parte de los/as estudiantes marroquíes realizaron su formación en centros de enseñanza españoles ubicados en Marruecos con lo cual estaban "obligados" a continuar la formación universitaria en España. Otro factor que explica la migración estudiantil de los/as jóvenes marroquíes es la generación de sistemas de redes socio-familiares que facilitan el proceso migratorio. De esta forma, aquellos/as que desean migrar para realizar estudios en España cuentan con amplia información sobre diferentes aspectos de la sociedad de acogida que obtienen de conocidos o familiares que ya experimentaron dicho proceso migratorio.

Por ultimo, otro factor presente en la elección de la Universidad de Granada por parte de los/as marroquíes es el endurecimiento producido en los requisitos necesarios para acceder a las universidades francesas, destino para muchos jóvenes marroquíes procedentes sobre todo del centro-sur de Marruecos ${ }^{3}$.

De forma paralela, la visión de retorno al país de origen tras la formación en España también alentó el proceso migratorio estudiantil ya que en muchos casos el único objetivo que explica la migración es la realización de una carrera universitaria en España que facilite la posterior incorporación al mercado laboral de Marruecos. Así, los estudios universitarios elegidos por los/as jóvenes marroquíes responden al desempeño liberal de la profesión una vez en su país. Por esta razón, en su mayoría realizan carreras universitarias tales como farmacia, medicina, traducción e interpretación. En este sentido, la Facultad de Farmacia desempeña un papel primordial, ya que en ella está matriculada la mitad de los marroquíes de la Universidad (aproximadamente de los 2000 estudiantes que cursan sus estudios en la Facultad de Farmacia, unos 600 son de nacionalidad marroquí según AEM). Después de farmacia, la elección de los/as estudiantes marroquíes apunta a las ingenierías superiores (144 en total agrupados en Granada, Valencia y Madrid), las licenciaturas en Ciencias (118 entre Granada y Barcelona) y medicina (97, de ellos más de la mitad en Granada) (López García, 1999). En cambio pocos son los que eligen carreras clásicas centradas en un empleo público como derecho, ciencias políticas, ciencias empresariales y carreras de enseñanza que les llevarían a incorporarse a la administración pública, es decir, a trabajar como dependientes del Estado en Marruecos. Cabe resaltar también que un 27 \% de los/as 
estudiantes marroquíes que residen en Granada preparan las pruebas de acceso a la Universidad en academias privadas (López García, 1999).

Dada la importancia, entre el colectivo de estudiantes marroquíes, que presenta el retorno al país de origen tras finalizar los estudios universitarios en España, en las páginas que siguen centraré el análisis en la fase de regreso a Marruecos así como la incorporación, tanto social como profesional, de los/as jóvenes a sus contextos de origen. Ello, no sin antes realizar algunas puntualizaciones sobre el propio alcance y significado del retorno.

10 A lo largo de los años de estancia de los/as estudiantes en la ciudad de Granada no son pocas las ocasiones en las que vuelven a sus ciudades de origen : en el transcurso de las vacaciones, para la celebración de las fiestas religiosas, etc. De tal manera que el movimiento de retorno está presente en todo el proceso migratorio de los/as estudiantes, desestimando así el carácter de fin de proceso que aparece muchas veces en la literatura sobre migraciones. A pesar de ello, el proceso de retorno que se analiza en este artículo tiene un carácter diferente al resto de posibles retornos o regresos ocurridos durante los años de estancia en la Universidad de Granada. Su peculiaridad radica en que dicho retorno se produce una vez finalizada la formación académica en España, realizándose con la intención de insertarse laboralmente en el mercado de trabajo de Marruecos, de ahí su carácter más o menos "definitivo» y no tanto transitorio, como ocurría a lo largo de los cursos académicos en años anteriores.

11 Cabe destacar que aunque he resaltado en los párrafos anteriores el retorno a Marruecos una vez concluidos los estudios universitarios, existen otras opciones escogidas por los/as estudiantes por las que el retorno no se produce y que es necesario también tener presentes. Entre ellas podemos destacar : el insertarse laboralmente en España, elegir otro país diferente al de origen para trabajar, planes de futuro con una pareja no marroquí, etc.

\section{Inserción profesional y reintegración socio-familiar en el país de origen}

Aunque las altas tasas de paro que presenta la sociedad marroquí dificultan la inserción profesional de las nuevas generaciones que buscan empleo, para aquellos marroquíes que han realizado sus estudios de farmacia, medicina o traducción, sobre todo, la dificultad que presenta la incorporación al mercado laboral sólo se refiere a la tramitación burocrática que se requiere para la apertura de la farmacia, de la consulta médica o del despacho. Así lo indicaba un joven tetuaní que realizó la carrera de farmacia en la Universidad de Granada y que estableció su farmacia en el pueblo de Mártil :

\footnotetext{
«pues me tiré un año corriendo para arriba y para abajo, no tenía dinero para montar la farmacia y pedí un crédito y claro todo el papeleo y tienes que convalidar tu título en el Ministerio de Educación y Ciencia y también en el de extranjeros y luego en el consulado marroquí y ya con todos los papeles, ya elegí el sitio y monté la farmacia » (Adel).
}

Por otra parte, la sociedad marroquí demanda especialmente los servicios que estas profesiones ofrecen, de manera que ayuda a la apertura de los mismos. A modo de ejemplo se puede citar el caso de las farmacias. En Marruecos, éstas son utilizadas al mismo tiempo como consulta médica ya que la mayor parte de la población marroquí 
no puede acceder a los costes que supone una visita médica más la compra de los medicamentos prescritos, de ahí que una farmacia solape las dos tareas respondiendo a una demanda primaria de la sociedad. Otro ejemplo es el desempeño de la traducción e interpretación en Marruecos, ésta tiene un amplio campo de ejecución ya que toda la tramitación necesaria para poder solicitar un visado y poder ir a España debe ser traducido por un traductor jurado y legalizado por el Consulado Español. En este sentido, un traductor de Al-hoceimas que tiene su despacho de traducción en dicha ciudad, hacía referencia a esta demanda social marcando la diferencia con España al respecto :

«la traducción aquí funciona mejor que en España porque compañeros míos españoles o marroquíes que se han quedado ahí pues no tienen este trabajo porque ese mercado no lo solicita, pero aquí sí porque como hay mucha gente que se quiere ir a España pues tienen que tramitar los papeles y sí hay gente que habla español pero no español jurídico o académico » (Mohamed).

Frente a los casos descritos anteriormente, donde el proceso migratorio responde a los objetivos que se plantearon los/as estudiantes en su inicio cumpliendo así las expectativas del proceso migratorio, existen otros muchos que, a pesar de realizar una fonación en el extranjero, no tuvieron una fácil imbricación en el sistema laboral de Marruecos.

Junto a la cuestión de la profesionalidad y la inserción profesional para el caso de los/as egresados/as marroquíes de la Universidad de Granada, existe otra variable importante de ser analizada : la aplicación de lo estudiado una vez que se encuentran desarrollando su labor profesional en el país de origen, y, en este sentido, las innovaciones incorporadas fruto de la experiencia formativa migratoria.

\section{¿Retorno y desarrollo?}

16 En el caso concreto de Marruecos y referente al proceso migratorio estudiantil de los/ as jóvenes marroquíes que se forman fuera de su país, podríamos suponer que el retorno de aquellos/as que finalizaron su formación y que ingresan en el mercado laboral del país constituyen un gran capital humano potencial de mejora, desarrollo, cambio y modernización del total del país. Sin embargo, ¿en qué medida esto se cumple?, ¿cuáles son los ámbitos en los que ingresan para promover el desarrollo?, ¿existe relación entre la formación adquirida y las necesidades actuales del país?

17 Durante la etapa migratoria, es decir, mientras trascurre la formación en España, son muchas las ocasiones en las que los/as estudiantes marroquíes reflexionan sobre los cambios posibles que pueden promover en el sistema marroquí una vez que finalicen la formación y regresen a su país. Así lo expresaba una estudiante procedente de la ciudad de Nador que realizaba su doctorado en Geología en la Universidad de Granada :

«siempre he pensado después de ver aquí como se trata a los alumnos y todo eso pues podría yo tomarlo de otra forma, también he tenido posibilidad de manejar muchos aparatos y entonces pues hay facultades que tienen ahora aparatos pero no todo el mundo los maneja, en este sentido me encantaría que me saliera en Marruecos porque yo te digo puedes aportar algo, tratar a los alumnos de otra forma que te han tratado a ti, dar las clases de otra forma, hacer más prácticas que teoría, mejorar las cosas, aplicar algo bueno que has aprendido aquí » (Rajja).

De tal manera que una vez que los/as jóvenes marroquíes se insertan laboralmente en Marruecos y según para qué disciplinas, las innovaciones desarrolladas dentro de cada 
sector son, en numerosas ocasiones, bastante visibles. Un médico traumatólogo tetuaní que se formó en la Universidad de Granada durante la década de los ochenta y que tiene su consulta privada en su ciudad natal, Tetuán, lo narraba así :

« se ha llegado a hacer cosas que no se habían hecho antes nunca, intervine en la primera prótesis total de cadera que se hace en el norte de Marruecos, los primeros clavos de Endel para el tratamiento de fractura de cadera en los ancianos, también las hernias de disco que no se hacían, y muchas primeras cosas » (Nader).

Sin embargo, a pesar de los conocimientos académicos que los marroquíes pueden transmitir a través de su trabajo, existe un enorme hándicap que limita su aplicación e innovación y que, por tanto, frena el desarrollo y la modernización que a través de este movimiento migratorio estudiantil se podría incorporar al funcionamiento estructural de Marruecos. Este hándicap es la falta de medios técnicos y recursos que permitan el desarrollo de determinadas tareas.

Como afirma Wood (1994), el desarrollo o la capacidad creciente de llevar una vida satisfactoria y de desarrollar el propio potencial, está claramente definido por las condiciones económicas, medioambientales, sociales y políticas a las que hacen frente las personas (1994: 16). Distintas sociedades, grupos e individuos podrán dar diferentes definiciones y pesos relativos a estas condiciones diversas en momentos concretos, pero todas ellas son importantes. Por ello, lo que se ha podido constatar a lo largo del trabajo de campo realizado en Marruecos, es que el optimismo que traen consigo los/as marroquíes que regresan a su país, por promover cambios y mejoras en el país se transforma en no menos que una clara resignación ante las dificultades técnicas y sociales de llevarlas a cabo.

\section{Retorno y reintegración familiar}

21 Por otra parte, junto a las dificultades presentes en el proceso de inserción profesional de los/as estudiantes en Marruecos tras el retorno migratorio se encontrarían también aquellas otras que se refieren a la reintegración socio-familiar en la sociedad marroquí. Frente a lo positivo de los años vividos en la migración existe lo complicado de la readaptación al contexto social-marroquí de origen tras la vuelta de España. En este sentido, son innumerables las situaciones que son fuente de conflicto y confrontación para estos/as jóvenes. Podríamos destacar varios ejemplos de ello según los datos obtenidos en las entrevistas realizadas así como del trabajo de campo realizado en Marruecos. Una de ellas es la que se refiere a las relaciones familiares y la convivencia de nuevo con la familia, donde vive la mayoría, tanto ellos como ellas, cuando regresan, ya que en la sociedad marroquí no se concibe la independencia de la familia si no estás casado/a o si el trabajo no requiere cambiar de ciudad de origen. De ahí que cuando los/as estudiantes marroquíes regresan a Marruecos tras finalizar la formación en España donde estuvieron viviendo de forma independiente encuentren dificultades en la convivencia familiar, como lo indicaba una joven farmacéutica de la ciudad de Larache :

« cuando volví pues me costó mucho pero muchísimo hasta convivir con mi familia, me cuesta bastante, porque yo me he acostumbrado a una vida de ahí que nadie, yo que sé, nadie me preguntaba a dónde vas o de dónde vienes » (Alma).

Este malestar que provoca volver de nuevo al ambiente familiar tras estar varios años fuera del mismo hace que tanto ellos como ellas promuevan cambios en su seno incorporando formas de comportamiento nuevas, fruto de la vivencia en España. Dichos 
comportamientos de alguna manera aprendidos durante la estancia fuera de Marruecos, corresponden a cotidianidades que pese a su simplicidad son apreciados en el entorno familiar, nos referimos a pautas de conducta en la mesa a la hora de la comida, al sistema de relaciones sociales que mantiene la familia, etc. Así lo indicaba un joven tetuaní que durante la década de los noventa estuvo realizando sus estudios de doctorado en Filología hispánica en la Universidad de Granada y que tras el retorno a su ciudad de origen pudo apreciar en el seno familiar los cambios producidos por su migración y la incorporación de nuevas formas de actuar ante situaciones que tal vez antes de la experiencia migratoria no eran sometidas a crítica alguna :

« cuando volví a mi casa la verdad es que me chocó el ambiente, la casa siempre llena de gente, me mosqueaba que no me gustaba mucho el ambiente, aquí ahora no invito a mucha gente, estás comiendo en el mismo plato y viene uno de la calle y viene y directamente mete la mano en el plato pues te da asco y me decían que yo si era malo y no sé qué pero son costumbres, ahora yo como en mi plato y Amal en el suyo y los vasos cada un con el suyo y eso en mi casa no es así, hay una jarra y un vaso y van echando y van bebiendo, la forma de vida de España, la trasladé un poco aquí » (Karim).

Otra cuestión que se incluye como fuente de choque en los/as estudiantes marroquíes tras el retorno es la que se refiere a la configuración de las relaciones sociales en torno a los sistemas de género. Tengo en cuenta que la formación del género (Thurén, 1993) responde a una construcción social y cultural en torno a los sexos y que, por tanto, no debe referirse exclusivamente a la visión de las mujeres, sino que debe integrar también al otro sexo. Pero me permito aquí hacer más hincapié en la vivencia que las mujeres tienen de su proceso migratorio y sus dificultades de re-adaptación a los códigos sociales marroquíes tras el retorno, ya que según el trabajo de campo realizado y mi propia estancia en Marruecos, en un porcentaje mucho más elevado, los problemas y el conflicto social y cultural están mucho más presentes en el retorno migratorio femenino que en el de sus compañeros masculinos.

De manera general, tanto el proceso migratorio como las fases estudiadas que lo integran se lleva a cabo de igual manera entre los y las jóvenes marroquíes que dejan temporalmente su ciudad marroquí de origen para dirigirse a una ciudad de acogida, Granada, para realizar estudios universitarios. Sin embargo, lo que no se presente de la misma forma es la visión femenina en cuanto « ser mujer » y su reflejo en las relaciones sociales generizadas (Ramírez, 1998).

\section{Relaciones de género tras el retorno}

El proceso de vuelta al entorno de la sociedad marroquí constituye un momento crucial después de una estancia prolongada y continua en España y esto es difícil, como se ha incluido en párrafos anteriores, tanto para los hombres como para las mujeres. Sin embargo, para ellas representa todavía más esfuerzo el adaptarse a las relaciones de género establecidas en la sociedad marroquí. Un ejemplo de ello lo constituye la distribución de los espacios sociales en función del sexo. En Marruecos la separación de sexos es fundamental en todos los ámbitos. Esta división de los espacios va a determinar, por supuesto, las relaciones sociales, lo cual no sucedía en los mismos términos durante su experiencia migratoria en la ciudad universitaria granadina.

Si en los testimonios de los/as estudiantes ya es frecuente la falta de espacios de ocio en Marruecos que ofrezcan alternativas para el tiempo libre en comparación con la 
sociedad granadina en la que permanecieron varios años, esto se acentúa para el caso de las mujeres :

« el ambiente de juventud y de movimiento, muchas veces aquí pues hay poco ocio, aquí si no lo buscas tú y haces tu ambiente y eso pues es difícil, allí salías y no te dabas cuenta del tiempo » (Ikram).

El espacio y el tiempo de ocio masculino transcurren en el café o en la calle directamente, es decir, lo más importante es la exteriorización de dicho tiempo (Ramírez, 1998). De esta forma, si para los hombres el tiempo transcurre en el espacio público para las mujeres ocurre todo lo contrario, el tiempo se interioriza en el ámbito de lo privado, lo cual no sería negativo en un primer análisis. Sin embargo, esto se convierte en algo denigrante en el momento en que las propias mujeres lo tachan de injusto y que, por tanto, las subordina y las limita en sus deseos más fundamentales. De esta forma, una joven tangerina licenciada y doctora en Biología celular por la Universidad de Granada, hace referencia a las actividades de las mujeres para su diversión, ubicadas siempre en el contexto privado y señala de esta forma su discrepancia al respecto :

«pues aquí ir a una boda para la gente de aquí es algo divertido pues para mí ponerme el kaftán, pintarme y arreglarme y pasar la tarde rodeada de mujeres para mí eso no es divertirme y la gente no lo entiende. Por ejemplo, en el trabajo, las chicas pues dicen que pasan las tardes en casa de una y otra y pasan la vida así, y yo no hago esto porque yo sé lo que es divertirme porque he vivido otra cosa y sé lo que es divertirse, yo casi no salgo a parte del trabajo no voy a ningún sitio » (Aicha).

Como se puede observar en el testimonio incluido anteriormente, la migración potencia la visión comparativa entre la forma de vida que se desarrolla en la sociedad de acogida y la presente en la de origen. Dicha visión es fuente generadora de malestar entre los/ as retornados/as ya que a menudo y respecto al tiempo de ocio en ambas sociedades, éste es criticado y analizado en función de la manera en como transcurrió el tiempo de diversión y ocio durante la estancia en España. De ahí que se critique, por ejemplo, la existencia de actividades claramente diferenciadas en función del sexo en la sociedad marroquí ya que no sucedió así durante la residencia en la ciudad de Granada. De esta forma, surge la comparación con un matiz negativo cuando se reflexiona sobre determinados aspectos de la sociedad marroquí. En este sentido, una joven farmacéutica de Tetuán que pasó ocho años en España lo señalaba así :

« hay pocos sitios para divertirse, para salir, pero bueno eso lo notas cuando te vas fuera y vuelves porque cuando estás al principio antes de salir pues no lo notas estás siempre acostumbrada a los amigos, la pandilla, la familia, y entonces te haces un mundo aquí y te acostumbras no sabes lo que hay pero una vez que sales fuera y te relacionas con gente y entonces esto después se te hace muy pequeño, cuando vuelves lo ves muy pequeño para lo que has vivido fuera " (Houda).

La construcción de un modelo cultural en torno al sexo y la explicación de éste en función de cuestiones biológicas existe en Marruecos, al igual que en otras sociedades, tanto para hombres como para mujeres. En el caso de las mujeres se instituye jurídicamente, por medio de la religión y de los textos sagrados, un modelo en el que son siempre "menores de edad ». Este modelo preconiza la dependencia de los menores respecto de los mayores : la de las mujeres respecto de los hombres (Ramírez, 1998). Así lo indicaba una joven tetuaní que trabaja como traductora en Larache y que tras casarse y divorciarse con un hombre español volvió a Marruecos para ejercer su profesión como traductora. En sus comentarios se puede apreciar la reivindicación de la mujer 
porque desaparezcan las limitaciones sociales que lleva impuestas por su condición de « menor » asociada a su condición de « mujer »:

«la mujer está totalmente limitada por su padre, su marido, su hermano porque imagínate que una mujer desde que nace hasta que muere es menor de edad, su padre es su tutor, si te casas es el marido y si no tienes ni padre ni marido pues es el hermano aunque éste sea más pequeño » (Somaya).

La condición de "eterna menor » hace que la mujer, para que tenga existencia social, deba necesariamente vincularse a un hombre. Dicha vinculación legitima la presencia de la mujer en el ámbito público de una forma más cómoda y tranquila. En este sentido, una médico que cursó sus estudios en Granada durante los años setenta y que se casó trascurrido poco tiempo tras el retorno a Marruecos después de finalizar sus estudios, resalta la situación de la mujer en la sociedad marroquí de aquella época de los setenta. Indica la existencia de lugares públicos vetados por su condición genérica de mujer y la necesaria presencia de un varón junto a ella en los lugares públicos :

« cuando volví en el 77 pues no podías sentarse en una cafetería, no puedes entrar al cine, con tu marido sí, a mí me ha dado la ventaja de haberme casado tan rápido después de venir entonces con tu marido sí puedes sentarte en una cafetería " (Houria).

31 Frente a la situación de la mujer en Marruecos hay casos entre los testimonios de los/as entrevistados/as que reivindican su no «necesaria » vinculación a un hombre para su realización personal en la vida social marroquí. Sin embargo, esta actitud crítica va unida, indiscutiblemente, al nivel socio-cultural de la mujer y a las posibilidades económicas con las que ésta pueda contar para su independencia personal, cosa que no siempre está presente en la vida de las mujeres marroquíes. Esta idea queda plasmada en el siguiente fragmento de una entrevista realizada a una joven farmacéutica de la ciudad rifeña de Al-hoceimas :

"buscar un marido por el hecho de tener un marido pues no, y claro encontrar a alguien que tenga una cultura parecida a la tuya pues no lo he encontrado, te encuentras con muchachas y te dicen jah ! pero no te has casado todavía, y el tema es ese, y dices, narices, mi vida no está supeditada a un hombre, al contrario yo quiero alguien que me compagine » (Fátima).

Como se observa en los testimonios de los/as profesionales entrevistados/as, un cambio también apreciable tras la migración se produce en lo referente al matrimonio y la forma de llevarlo a cabo en el contexto de origen. La posible existencia de un pacto social y familiar sin tener en cuenta el deseo de los futuros contrayentes a la hora de establecer un matrimonio, es rechazado por los/as retornados/as. A su vez, éstos incitan nuevas formas de llevarlo a cabo y bajo otros criterios muy diferentes que no responden a las condiciones de dicho " pacto socio-familiar » :

« todavía no estoy del todo adaptada, estoy aquí pero al mismo tiempo ahí, porque estás aquí pero yo no me meto en el mundo de por ejemplo de aquí de ir de visitas a las casas y hacer la vida de señorita que hacen aquí o plantearme casarme así sin conocer al hombre de mi vida, así lo hacen aquí » (Fatija).

Por otra parte, el hecho de la necesaria existencia de un hombre como punto de referencia en la vida de las mujeres, limita y condiciona, en la mayoría de los casos, la forma de vestir de éstas. Si en Marruecos no se concibe fácilmente que una mujer lleve un vestido determinado que marque su forma del cuerpo, esto empeora cuando se trata de una mujer casada. En este sentido, una bióloga tangerina que trabaja en el Sistema Sanitario de Tetuán indica la diferencia en el vestir tras casarse con su marido, ello impuesto socialmente : 
«allí (España) ponía vaqueros, iba a la discoteca, ponía tops, ahora no puedo ponerme vaqueros porque estoy casada y la gente te mira (...), no puedes comportarte como allí, jamás he puesto vaqueros a partir de casarme porque aquí una chica casada no tiene que ponerse vaqueros, y además la gente no te va a dejar tranquila te dicen piropos malos ni siquiera como los españoles que te dicen guapa, morena, no sé qué, nada más que las cosas feas que no quieres oír, tienes que ponerte la ropa tapándote el culo. Si estás casada no te permiten nada. No pongo yilaba ni pañuelo en la calle pero siempre voy tapada » (Loubna). hombres, es algo que aparece con mucha frecuencia en el discurso de las mujeres entrevistadas para esta investigación. Ello es resaltado como una diferencia importante entre la estancia en España y el día a día del retorno a Marruecos. El estar en el extranjero supone estar fuera de la presión social del contexto de origen que imponía una forma de estar para con los demás que rechaza la naturalidad, sobre todo para las mujeres. Para gran parte de la sociedad marroquí las mujeres que migraron y que vivieron fuera de Marruecos son portadoras de valores contrarios a los tradicionales marroquíes tales como el honor, la virginidad, la respetuosidad, etc., y no sólo esto ocurre para las mujeres marroquíes sino también para las mujeres extranjeras cosa que pude observar durante mis estancias en Maruecos. Una mujer extranjera en Marruecos es percibida como una mujer liberal donde no hay límite alguno en una relación con un hombre, aunque se trate simplemente de una relación de amistad. Ésta no es percibido de esta forma en la sociedad marroquí donde no se concibe la existencia de una pura amistad entre un hombre y una mujer. Esta forma de entender las relaciones es fuente de conflicto para las mujeres marroquíes tras el retorno ya que a menudo, son vistas de la misma forma que una extranjera. Esta actitud para con las mujeres no proviene tanto de los hombres que migraron y vivieron la experiencia migratoria sino de aquellos que nunca salieron del país ya que éstos no vivieron en otro modelo cultural. Esta situación establece límites que las propias mujeres desarrollan cuando se relacionan con los hombres marroquíes. Es reflejo de ello el siguiente testimonio de una farmacéutica que se formó en la Universidad de Granada durante la década de los 80 y que actualmente tiene su farmacia en la ciudad de Al-hoceimas :

« de ahí (España) pues vienes con una soltura, en forma de vestimenta y eso y te empiezan a molestar y además la idea, salvo la gente que ha estudiado fuera la carrera y tal pues te ven... la gente que no ha estado fuera pues te ven como una chica liberal porque hayas estudiado fuera cinco años fuera de tu casa, que a lo mejor eres una chica fácil (...) y empiezas a hablar y a reírte con la gente y empiezan a faltarte el respeto creyendo que eres una chica fácil por eso al comportarse de esa forma con nosotros pues ya pones tus límites y cambias de carácter » (Chumisa).

La interpretación que tiene para la sociedad marroquí el comportamiento "natural » de una mujer obliga a muchas mujeres marroquíes a actuar interpretando "papeles dobles ». Atienden, así, a la división entre el ámbito privado y el público y entre vivir en el extranjero y estar en las respectivas ciudades marroquíes. La diferencia, de manera general, radica en que en el primer caso la mujer puede comportarse como ella misma y en el segundo caso, es decir, estando en Marruecos, el comportamiento de la misma debe responder al patrón establecido en la sociedad marroquí que diferencia espacios sociales según sexo $\mathrm{y}$, con ellos, formas de comportarse. Así lo expresaba una farmacéutica de Al-hoceimas :

« cambia la mentalidad totalmente, te cambian muchas cosas, la manera de pensar,

la manera de vivir también, ahí ven a una mujer que tiene derechos, la mujer aquí no tiene derecho tiene que estar en casa, bueno yo no hablo de todos pero hablo de 
la mayoría, la mayoría son así. Aquí es como un papel que estamos interpretando, pensamos de una forma y nos comportamos de otra, mi marido también me lo dice, cuando estamos en España haz lo que te de la gana pero aquí ya sabes que no y no es por él, es por la gente » (Yamila).

Como se ha comprobado a lo largo de estas páginas, el retorno de los/as jóvenes marroquíes a su contexto de origen va acompañado de innumerables contradicciones, reajustes y readaptaciones a todo lo referente a los códigos sociales, familiares y morales presentes en la sociedad marroquí. En todo ello, las relaciones sociales en base a la construcción de género ocupan un lugar primordial en la dificultad de insertarse de nuevo en Marruecos tras vivir varios años fuera de dicho país.

Por último, quiero resaltar que no ha sido mi intención expresar una imagen estereotipada de Marruecos y de las mujeres marroquíes en comparación con otros patrones socioculturales, sino más bien, reflexionar sobre las dificultades de readaptación al contexto de origen que presentan las jóvenes marroquíes tras la experiencia migratoria vivida, esto es a través de sus propias vivencias, discursos y sensaciones.

\section{A modo de conclusión}

Desde un punto de vista antropológico, el proceso migratorio de los estudiantes marroquíes se inscribe dentro del análisis de los movimientos migratorios contemporáneos y las relaciones interculturales, a pesar de que se trata de un tipo especifico de proceso migratorio cuyo origen responde a motivos que no son $\mathrm{ni}$ familiares, ni económicos... En el caso del proceso migratorio de los/as estudiantes marroquíes, el estudio del sistema de redes migratorias, que apoya y forja el proceso migratorio, y la transnacionalidad de dichas redes que evita la visión de las migraciones como un proceso unidireccional dirigido desde una sociedad de origen dada hacia una sociedad de acogida determinada, se presenta como imprescindible.

39 Al mismo tiempo, la visión de retorno al país de origen que presentan los/as estudiantes marroquíes desde el inicio del proceso migratorio, hace que también sea necesario el estudio del mismo. Dicho retorno al contexto de origen tras la realización de los estudios universitarios en España va acompañado de innumerables contradicciones, reajustes y readaptaciones a todo lo referente a los códigos sociales, familiares y morales presentes en la sociedad marroquí. El retorno se presenta así como un proceso con dificultades tanto profesionales como personales. En primer lugar, la búsqueda de un empleo por parte de los/as retornados/as en la sociedad marroquí acorde con las expectativas iniciales personales no siempre es fácil y, por otro lado, la reinserción socio-familiar en el contexto de origen tras la experiencia migratoria también se convierte en un proceso arduo en el que se requiere un considerable tiempo de readaptación.

Por otra parte, se puede observar que ciertas de las experiencias y calificaciones adquiridas por los migrantes no son movilizables a corto plazo para favorecer el desarrollo del país de origen. Por una parte, no existen buenas condiciones para la inversión en el país de origen y, por otra parte, no siempre las formaciones adquiridas responden a las necesidades locales de la sociedad de origen. Sin embargo, a pesar de que no existe una estructura gubernamental y unas infraestructuras adecuadas, habría que valorar los efectos de la migración estudiantil sobre el desarrollo de Marruecos a 
distintas escalas (Lacomba, 2002) : internacional, nacional, regional, local, familiar e individual, así como también a corto, medio y largo plazo. El capital que consiste, en este caso, en la formación adquirida, en sentido amplio por los/as jóvenes marroquíes en el extranjero se convierte en un fuerte motor de desarrollo de diferentes ámbitos y sectores del país de origen pero su incorporación debe ser lenta y gradual.

\section{BIBLIOGRAFÍA}

AGUIRRE BAZTÁN Ángel (1995) Etnografía. Metodología cualitativa en la investigación sociocultural, Barcelona, Marcombo, $356 \mathrm{p}$.

BERNARD H. Russel Éd (1988) Handbook of Methods in Cultural Anthropology, Walnut Creek, California, AltaMira, $520 \mathrm{p}$.

GEISSER Vincent y LATRECHE Abdelkader (2001), Faire ses études en France, et après ? Le devenir des diplômés maghrébins formés dans l'Hexagone, Migrations Societé, vol. 13, n 74, pp. 87-97.

GONZÁLEZ BAREA Eva María (2000) Hacia una comunidad transnacional ? El proceso migratorio de los estudiantes marroquíes en Granada, Departamento de Antropología y Trabajo Social, Universidad de Granada, $108 \mathrm{p}$.

LACOMBA VÁZQUEZ Joan (2002) Los efectos de la emigración sobre el desarrollo. El caso de las migraciones en Marruecos, in García Castaño, F.J. y C. Muriel López eds., La emigración en España : contextos y alternativas, Volumen II. Actas del III Congreso sobre la inmigración en España (Ponencias), Granada, Laboratorio de Estudios Interculturales, pp. 469-480.

LÓPEZ GARCÍA Bernabé (1999) El intercambio universitario entre Marruecos y España : los estudiantes marroquíes en España. Awraq, XX : pp. 233-267.

RAMÍREZ ángeles (1998) Migraciones, género e islam. Mujeres marroquíes en España, Madrid, AECI, 375 p.

SIMON Valérie (1992) Les étudiants maghrébins à l'Université en France, 1962-1991, Mémoire de DEA, «Connaissance des Tiers Mondes », Université Paris VII, UFR G.H.S.S., 53 p.

SLAMA Serge (1999) La fin de l'étudiant étranger, Paris, L'Harmattan, 312 p.

THURÉN Britt. Marie (1993) El poder generizado. El desarrollo de la antropología feminista, Madrid, Instituto de investigaciones feministas y Dirección General de la mujer, 115 p.

WOOD Bernard (1994) Estrategias de desarrollo y emigración : vinculaciones y posibles lecciones, Desarrollo, 24, pp. 15-24.

\section{ANEXOS}




\section{Metodología del proceso de investigación}

Para esta investigación se han realizado amplios periodos de trabajo de campo estableciendo una relación directa con el grupo que ha protagonizado el proceso migratorio analizado. Durante dichos periodos, tanto la perspectiva de estudio emic como la etic se han entrelazado y han estado así presentes en numerosas ocasiones.

Tras un primer acercamiento a las fuentes bibliográficas así como un breve contacto previo con los/as estudiantes marroquíes instalados en la ciudad de Granada, se llevó a cabo la primera estancia en Marruecos que fue realizada en el verano de 1999. Este primer contacto con el país aportó un conocimiento imprescindible para continuar la investigación, una oportunidad para conocer de una forma cercana la realidad marroquí y los aspectos culturales de la misma, sus fiestas y sus formas de vida cotidiana.

Tras ese primer contacto con Marruecos y contexto de origen de los estudiantes comenzó la fase de recopilación de datos propiamente dicha en la ciudad de Granada. Se estuvieron realizando entrevistas a los/as estudiantes marroquíes durante su fase migratoria, su periodo de formación universitaria. Ésta fue tratada y analizada, realizando una primera redacción sobre dos de las fases del proceso migratorio estudiantil analizado, la fase pre-migratoria y migratoria del proceso investigado.

Posteriormente, se realizó una estancia larga en Marruecos para poder estudiar, por una parte, el retorno de estos jóvenes marroquíes y, por otra parte, el inicio del proyecto migratorio de aquellos estudiantes marroquíes que comenzaban su proceso migratorio hacia España.

Para ello, desde noviembre del año 2000 hasta agosto del 2001 estuve viviendo en Mártil, una localidad cercana a Tetuán. La elección de dicha zona de residencia responde a la proximidad que me permitía en el acceso a los informantes ya que el norte constituye, en términos cuantitativos, el mayor proveedor de la salida de estudiantes marroquíes hacia la Universidad de Granada y por tanto, por franja temporal, el mayor número de retornados.

Durante este periodo se realizaron entrevistas a los ex - estudiantes de la Universidad de Granada, así como a las distintas instituciones educativas españolas, ya que la mayoría de los/as estudiantes marroquíes que deciden proseguir sus estudios en España y, en concreto, en Granada, iniciaron sus estudios en centros españoles ubicados en Marruecos. También se recopiló información a través de distintas academias de lengua española presentes en Marruecos, así como de centros consulares de España, que ofrecieron información sobre el aspecto legislativo de la estancia y residencia de los/as estudiantes en España.

Tras este periodo prosiguió el análisis y redacción de la información recopilada manteniendo una distancia necesaria para el análisis de la misma. Una vez en Granada, también prosiguió el trabajo de recopilación de datos referente a los/as ex estudiantes marroquíes de la Universidad de Granada que decidieron no regresar al país de origen tras finalizar su estancia de estudio en España.

Los grupos de la muestra de estudio se han ido configurando a medida que nos hemos ido adentrado en su estudio. Se han realizado un total de sesenta y una entrevistas semi estructuradas ( 32 mujeres y 29 hombres) referentes a la realidad de los/as estudiantes y 
ex estudiantes marroquíes de la Universidad de Granada y el transcurso de su proceso migratorio. El guión de la entrevista que se ha utilizado para esta investigación incluye tres bloques de temas, que se pueden agrupar en un continuo temporal (antes de salir de Marruecos, estancia en España y retorno y planes de futuro en el país de origen). A su vez, cada uno de los bloques del guión incluye preguntas que quedan divididas en tres ámbitos temáticos : educación, familia y relaciones sociales. Ellos/as mismos/as la han definido a través del trabajo de campo realizado entre el propio colectivo de estudiantes, estableciendo redes sociales de informantes que, a su vez, han establecido redes de conocidos que han pasado fronteras y que en numerosas ocasiones nos han llevado de Granada a Marruecos y viceversa. Con ello, se ha seguido un procedimiento consciente, exploratorio y paulatinamente focalizado de muestreo, el denominado «judgement sampling» (Bernard, 1988).

\section{NOTAS}

1. Este trabajo forma parte de mi tesis doctoral «El proceso migratorio de los/as estudiantes marroquíes a la Universidad de Granada. ¿Hacia una comunidad transnacional ? », realizada dentro del Departamento de Antropología y Trabajo Social de la Universidad de Granada y defendida en septiembre del año 2003.

2. Recogido en Gaceta Universitaria de Granada, 14 de febrero de 2000.

3. Sobre la realidad de los/as estudiantes marroquíes en Francia ver Geisser y Latreche, 2001 ; Simon 1992 y Slama 1999.

\section{RESÚMENES}

La investigación presentada aquí analiza un proceso migratorio particular al mismo tiempo que se incluye una novedosa y poco estudiada noción de migración. Describe el desplazamiento de estudiantes procedentes de distintas regiones de Marruecos para realizar su formación académica en una universidad extranjera y, en concreto, en la Universidad de Granada. A pesar de su diferencia, incluye a esta migración dentro del fenómeno global de las migraciones contemporáneas.

El articulo se interesa particularmente a los/as estudiantes/as que han terminado sus carreras y que retornan a Maruecos. Describe, a partir del discurso de los interesados, sus aspiraciones a participar al desarrollo de su país, y las dificultades de su reinserción en la sociedad marroquí, tanto en el ambito rofesional como en la vida familiar y social.

Les étudiants marocains en Espagne : migration, retour et genre

La recherche présentée dans cet article analyse un processus migratoire particulier en même temps qu'elle enrichit la notion peu étudiée de migration étudiante. Elle décrit le déplacement d'étudiants provenant de diverses régions du Maroc pour poursuivre leurs études et acquérir un diplôme dans une université étrangère, ici celle de Grenade, en Espagne. Elle replace cette migration, malgré ses caractères particuliers, dans le phénomène global des migrations contemporaines. 
L'article s'intéresse en particulier aux diplômés/ées rentrant au Maroc, décrivant à partir de leur propre discours leurs aspirations à contribuer au développement de leur pays et leurs difficultés à se réinsérer dans la société marocaine, tant dans le domaine professionnel que dans la vie familiale et sociale.

The Moroccan Students in Spain : Migration, Return and Gender

In this article we analyse a particular migratory process. We also include a new approach of the neglected notion of student migration. The research consists of the displacement of students of different regions of Morocco to continue their academic formation in a foreign university, in this case, the University of Granada. We present the study as a new research topic, due to the lack of studies in this matter.

This article presents the analysis of a new migratory process that we necessarily have to include in the global phenomenon of actual migrations. It describes the aspirations of the students to contribute to the development of their country and the difficulties to be inserted in the Moroccan society from a professional and social point of view.

\section{ÍNDICE}

Índice geográfico: Espagne

Mots-clés: élite, étudiants, Marocains, réinsertion au pays d'origine, retour

\section{AUTOR}

\section{EVA MARÍA GONZÁLEZ BAREA}

Universidad de Granada, Dpto. de Antropologia y Trabajo Social, Facultad de Ciencias de la Educación E-18071 Granada (Spain), tel. : +34-958-24 63 43, E-Mail : evamgb@ugr.es Web:www.ugr.es/ ldei/. 